\title{
Estudio comparativo de la utilización de ancho de banda con los protocolos SIP e IAX
}

\section{A comparative study of bandwidth usage running protocols SIP and IAX}

\section{OCtavio Salcedo}

Ingeniero en Sistemas, magister en Teleinformática, candidato a Doctor en Informática. Docente de la Universidad Distrital Francisco José de Caldas. Bogotá, Colombia. Contacto: ojsalcedop@udistrital.edu.co

\section{Danilo López}

Ingeniero Electrónico, magíster en Teleinformática. Docente e investigador de la Universidad Distrital Francisco José de Caldas. Bogotá, Colombia.

Contacto:dalopezs@udistrital.edu.co

\section{Cesar Hernández}

Ingeniero Electrónico, magister en Ciencias de la Información y las Comunicaciones, estudiante de doctorado en Ingeniería de Sistemas y Computación de la Universidad Nacional de Colombia. Docente e investigador de la Universidad Distrital Francisco José de Caldas. Bogotá, Colombia.

Contacto: cahernandezs@udistrital.edu.co

Fecha de recepción: 13 de noviembre de 2011

Fecha de aceptación: 28 de agosto de 2012
Clasificación del artículo: Revisión de Tema

Palabras clave: ancho de banda, audio, codec, IAX, SIP, VoIP.

Key words: bandwidth, audio, codec, IAX, SIP, VoIP.

\section{RESUMEN}

Dentro de los ambientes de voz sobre IP, existen tres protocolos que resuelven el problema de la señalización de paquetes de voz. A estos protoco- los se les conoce con el nombre de "highlight protocols" (protocolos de realce) y son: H323, SIP e IAX. Este artículo se enfoca, particularmente, en una de las diferencias específicas entre SIP e IAX: el uso del ancho de banda, que es un pará- 
metro esencial al momento de diseñar y optimizar una red VoIP para una compañía. Uno de los factores más importantes que se deben considerar al momento de construir redes VoIP es la capacidad de planeación adecuada. Con respecto a la capacidad de planeación, el cálculo del ancho de banda es un factor importante que se debe considerar al momento de diseñar y solucionar problemas en redes de voz empaquetada; de manera tal, que se garantice calidad en la voz. Este artículo brinda explicaciones importantes acerca del uso del ancho de banda sobre las redes VoIP. Se incluyen fundamentos teóricos y cálculos de ancho de banda por flujo para una troncal VoIP, en la cual se habilitan varios codecs de voz (específicamente G.711 y GSM) junto con un protocolo VoIP tal como SIP e IAX.

\section{ABSTRACT}

Within the VoIP networks environment, there are three protocols that solve the problem of voice packet signaling, known as "highlight protocols": H323, SIP and IAX. Particularly, this document focuses on a specific difference between SIP and IAX: the bandwidth usage, which is an essen-

\section{INTRODUCCIÓN}

En los modelos de la tecnología VoIP, los protocolos SIP, IAX, H.323 y RTP se encargan de la señalización y la transferencia de media. En este estudio, se realiza una comparación entre SIP e IAX con base en uso de ancho de banda (factor fundamental a la hora de escoger el esquema de comunicación para troncales VoIP), teniendo en cuenta: 1) los requisitos básicos para una aplicación de audio conversacional [1],2) los posibles codecs de audio a utilizar [2], 3) el overhead en conversaciones VoIP causado por los protocolos involucrados en la comunicación [3] y 4) el aná- tial parameter in order to design and optimize a VoIP network for an organization. One of the most important factors to consider when building VoIP networks is proper planning capacity. About planning capacity, bandwidth calculation is an important factor to consider when designing and troubleshooting packet voice networks for good voice quality. This paper presents important explanations about the bandwidth utilization over VoIP networks; this includes some theoretical foundations and bandwidth calculation on a perflow basis for a VoIP trunk, in which different voice codecs are enabled (specifically G.711 and GSM) together with VoIP protocols such as SIP and IAX. Voice over IP (VoIP) is applied. These calculations are compared against the results obtained from traffic analyzers over a physical Ethernet trunk between two Asterisk servers into a specific LAN, simulating two different branches of an organization. Both the analysis of the results obtained and the conclusions from this work are useful when calculating the maximum number of simultaneous calls or the minimum capacity of a data link that is necessary for a particular number of voice conversations, taking into account the audio codecs used and the signaling and data flow protocols.

\section{$* *$}

lisis del formato de las tramas SIP e IAX con su respectivo encapsulado [4].

En el presente trabajo, se aplicarán los fundamentos anteriores para realizar cálculos teóricos de consumo de ancho de banda, comprobando lo anterior, experimentalmente, por medio de la implementación de una troncal física que sirva de enlace entre dos servidores de diferentes segmentos de red dentro de una LAN; con su correspondiente diseño arquitectónico y análisis de tráfico en producción, simulando cierto número de llamadas simultaneas sobre los protocolos SIP (el cual usa el protocolo RTP para 
flujo de media) e IAX, para un numero definido de conversaciones. Para tal fin, se realiza la configuración de los routers y de las centrales telefónicas y se utilizan analizadores de protocolos para obtener el valor de ancho de banda utilizado por la red real [5].

Finalmente, se plantean las conclusiones más relevantes, que serán un marco de referencia útil en el momento de diseñar, dimensionar y optimizar una red VoIP (pudiéndose escalar este estudio a redes WAN con protocolos y arquitecturas tales como PPP, ATM y Frame Relay), utilizando los codecs de audio y los protocolos de señalización y de flujo de media adecuados para cada escenario especifico.

\section{PROTOCOLOS VOIP}

Los protocolos VoIP pueden agruparse en dos categorías generales: centralizadas y distribuidas [6]. Puntualmente, la arquitectura distribuida de redes VoIP está asociada, principalmente, con los protocolos H.323, SIP y el reciente IAX. Estos protocolos permiten que la inteligencia de la red se distribuya entre dispositivos de control de llamadas y dispositivos finales (Gateways VoIP, teléfonos IP, servidores media, o cualquier dispositivo que pueda iniciar y terminar una comunicación VoIP).

\subsection{Modelo arquitectónico de los protocolos VolP}

Como punto de referencia desde el punto de vista estructural, la figura 1 muestra la distribución de los protocolos utilizados en VoIP dentro del modelo OSI.

Por sus cualidades de simplicidad y bajo overhead, en sus formatos respectivos con respecto al protocolo H.323, este trabajo se enfocará en

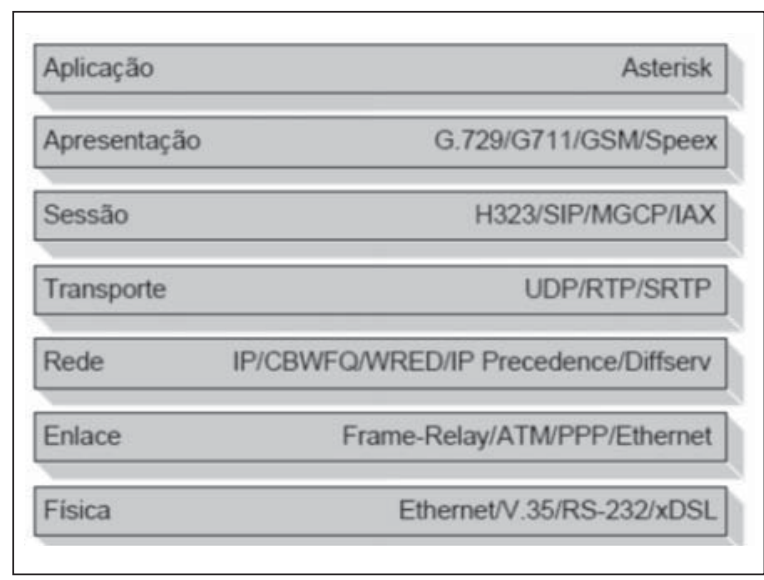

Figura 1. Modelo arquitectónico de los protocolos de VolP dentro del modelo OSI. Fuente: tomado de [3]

los protocolos SIP e IAX, los cuales, como se ve en la figura, son protocolos de inicio de sesión.

\subsection{Protocolo SIP}

SIP (Session Iniciation Protocol) es un protocolo de señalización de la capa de sesión del modelo OSI, desarrollado y estandarizado por la IETF (razón por la cual su adopción en el mercado es evidente) para establecer, configurar, modificar y finalizar sesiones multimedia [7]. Al igual que otros protocolos de dicha capa (como HTTP), SIP se basa en el mecanismo de "petición y respuesta" (request/response) para iniciar una sesión de comunicación. Este protocolo permite establecer sesiones para transmisión de datos $\mathrm{y}$ video entre dos puntos finales, definiendo los sockets y la negociación de los tipos de archivos y los formatos [1], [3], [4].

Una vez la sesión es establecida, la responsabilidad de la transferencia del flujo de media, en el caso de voz y video, en tiempo real, es delegada al protocolo RTP, el cual asigna dinámicamente un puerto, normalmente perteneciente al rango 10000-20000 [8]. 


\subsection{Protocolo IAX}

Inter-Asterisk eXchange Protocol es un protocolo abierto de Asterisk, ratificado con la RFC 5456; proporciona control y transmisión de voz sobre redes IP [6]. Implementado en principio para 1lamadas de voz, actualmente puede ser usado con cualquier tipo de media como voz y video. Los dos objetivos principales del proyecto IAX derivarán de la experiencia con los protocolos de VoIP como el SIP y el MGCP (Media Gateway Control Protocol) para control y el RTP y flujo multimedia y son [3]:

- La minimización del uso de ancho de banda, tanto para el tráfico de control como el de media, con énfasis específico en conversaciones de voz.

- Proveer transparencia a NAT.

IAX es un protocolo de media y señalización punto a punto, cuyo fundamento es el de la multiplexación de la señalización y múltiples flujos sobre una única asociación UDP entre dos usuarios finales [9].

Asimismo, IAX utiliza el puerto UDP 4569 para comunicar todos los paquetes, usando para tal fin un campo de llamada de 15 bits dentro de la cabecera del protocolo, para multiplexar los flujos sobre una única asociación UDP [3].

La implementación conocida como IAX Trunked permite que el protocolo use una única cabecera para el pasaje de varias llamadas, es decir, que es posible enviar varias conversaciones por el mismo flujo, lo cual supone un importante ahorro de ancho de banda, ya que con esto se evita el overhead de las capas IP y transporte UDP excesivo. Ahora bien, hasta el momento el modo IAX trunked sólo puede ser habilitado entre dos servidores Asterisk.
A continuación se realiza la comparación, basados en el uso de ancho de banda, de los dos protocolos mencionados.

\section{CÁLCULO DEL USO DE ANCHO DE BANDA EN REDES VOIP}

\subsection{Encabezados de protocolos de capas inferiores OSI}

De acuerdo con [2], los tamaños de encabezado de los protocolos que intervienen en la señalización y el flujo de paquetes de voz son mostrados en la tabla 1. Dichos encabezados no pueden ignorarse, ya que son indispensables para el flujo de media:

Tabla 1. Tamaños de encabezados de protocolos utilizados.

\begin{tabular}{|l|l|l|}
\hline PROTOCOLO & NIVEL OSI & CABECERA \\
\hline Ethernet & Enlace de datos (2) & 18 Bytes \\
\hline IP & Red (3) & 20 Bytes \\
\hline UDP & Transporte (4) & 8 Bytes \\
\hline
\end{tabular}

Fuente: tomado de [10]

De acuerdo con parámetros como el equilibrio entre la calidad de voz y el consumo de ancho de banda, se escogen, para este estudio, los codecs G.711 y GSM por su calidad y su bajo consumo, respectivamente [1], [3].

\subsection{Overhead en conversaciones VolP causa- do por los protocolos de comunicación}

En una red VoIP, el ancho de banda de la conversación no está dado solamente por la carga que aportan los métodos de compresión y codificación de audio (codecs); se tiene asimismo una sobrecarga causada por las cabeceras de los protocolos de las capas de enlace, red y transporte del modelo TCP/IP [8], [10]. De esta forma, se 
puede decir que la necesidad de ancho de banda varía de acuerdo con los tipos de cabecera involucrados en el paquete. Por ejemplo, al estar en una red Ethernet, se debe adicionar el encabezado Ethernet - capa de enlace- al cálculo. Igualmente, al estar en una red WAN se debe sumar el encabezado PPP, ATM o Frame Relay, según el caso, lo cual representa un incremento significativo de la cantidad de banda utilizada.

\subsection{Parámetros requeridos para el análisis del uso del ancho de banda}

- Codec Sample Interval (CSI) (ms)

- Codec Sample Size (CSS) (Bytes)

- Codec Bit Rate (CBR) (Kbps): se calcula con la siguiente expresión:

$$
C B R=C S S / C S I
$$

- Voice Payload Size (VPS) (bytes ó ms)

- Packets Per Second (PPS): se calcula con la siguiente expresión:

$$
P P S=C B R / V P S
$$

- Total Packet Size (TPS) (bytes): está dado por la expresión:

$$
T P S=L 2+I P+U D P+L 5+V P S
$$

VPS: El VPS no corresponde al tamaño total del paquete de voz (Total Packet Size o TPS) que transita por la red VoIP; es necesario incluir los encabezados de los otros protocolos involucrados en la transmisión, correspondientes a las capas inferiores del modelo.

L2: Tamaño de la cabecera del protocolo de la capa de enlace de datos (Ethernet, Frame Relay, PPP)

$I P$ : Tamaño de la cabecera IP
UDP: Tamaño de la cabecera UDP

L5: Tamaño de la cabecera del protocolo de sesión (H323, SIP/RTP o IAX)

- Bandwidth (BW) (Kbps): el ancho de banda, del canal, necesario es: para $n$ conversaciones full duplex (el coeficiente 2 que aparece en la expresion se debe a que una conversacion requiere dos flujos de informacion (full $d u$ plex).):

$$
B W n=B W \times n \times 2
$$

\subsection{Cálculo BW con protocolo SIP-codec G.711}

Datos de entrada:

- $C S I=10 \mathrm{~ms}$

- $C S S=80$ Bytes

- $V P S=160$ Byte

$$
\begin{aligned}
& C B R=\frac{C S S}{C S I} \\
& C B R=\frac{80 \text { Bytes } * 8 \frac{\text { bits }}{\text { Byte }}}{10^{-2} s} \\
& C B R=64 \mathrm{Kbps}
\end{aligned}
$$

Como el $C S S=80$ Bytes es capturado en un intervalo CSI de $10 \mathrm{~ms}$, el cálculo del VPS ( $\mathrm{ms}$ ) para 160 Bytes (que corresponde al tamaño del VPS para dicho codec) se obtiene de una sencilla regla de tres:

$\mathrm{CSS} \rightarrow \mathrm{CSI}$

$\mathrm{VPS} \rightarrow$ VPS (ms)

Luego,

$$
\operatorname{VPS}(m s)=\frac{V P S * C S I}{C S S}
$$




\section{revisión |}

$$
\begin{gathered}
V P S(m s)=\frac{160 \text { Bytes } * 10 \mathrm{~ms}}{80 \text { Bytes }} \\
V P S(m s)=20 \mathrm{~ms} \\
P P S=\frac{C B R}{V P S} \\
P P S=\frac{64 \text { kbps }}{160 \text { Bytes } * 8 \frac{\text { bits }}{\text { Byte }}}
\end{gathered}
$$

- $\quad \mathrm{PPS}=50$

Para el cálculo del TPS, Los tamaños de las cabeceras de protocolos inferiores se toman de la tabla 1 (el montaje se va a implementar sobre Ethernet)

$$
\begin{gathered}
T P S=L 2+I P+U D P+L 5+V P S \\
T P S=18 \text { Bytes }+20 \text { Bytes }+8 \text { Bytes }+ \\
12 \text { Bytes }+160 \text { Bytes }
\end{gathered}
$$

- $T P S=218$ Bytes $=1744$ Bits

$$
\begin{gathered}
B W=T P S \times P P S \\
B W=1744 \text { Bits } \times 50
\end{gathered}
$$

- $B W=87.2 \mathrm{Kbps}$

Para 30 conversaciones:

- $B W 30$ calls $=87.2 \mathrm{Kbps} \times 30 \times 2=5232 \mathrm{Kbps}$ (más de 5Mbps)

\subsection{Cálculo BW protocolo SIP - codec GSM}

Datos de entrada:

- $C S I=20 \mathrm{~ms}$

- $C S S=33$ Bytes

- $V P S=33$ Bytes

$$
C B R=\frac{33 \text { Bytes } * 8 \frac{\text { bits }}{\text { Byte }}}{20 * 10^{-3} \text { s }}
$$

- $C B R=13.2 \mathrm{Kbps}$

$$
\operatorname{VPS}(m s)=\frac{V P S * C S I}{C S S}
$$

$$
\operatorname{VPS}(\mathrm{ms})=\frac{33 \text { Bytes } * 20 \mathrm{~ms}}{33 \text { Bytes }}
$$

- $\operatorname{VPS}(\mathrm{ms})=20 \mathrm{~ms}$

$$
P P S=\frac{C B R}{V P S}
$$

$$
P P S=\frac{13,2 \text { kbps }}{33 \text { Bytes } * 8 \frac{\text { bits }}{\text { Byte }}}
$$

- $P P S=50$

$$
\begin{aligned}
& T P S=L 2+I P+U D P+L 5+V P S \\
& \text { TPS }=18 \text { bytes }+20 \text { Bytes }+8 \text { Bytes }+12 \text { Bytes } \\
& +33 \text { Bytes } \\
& \text { - } \quad T P S=91 \text { Bytes }=728 \text { Bits } \\
& B W=T P S x P P S \\
& B W=728 \text { Bits } x 50
\end{aligned}
$$

\subsection{Protocolo IAX-Codec G.711}

En el caso particular de IAX, cuando más de una llamada es hecha, el overhead de los encabezados IP es disminuido, encaminando múltiples paquetes de voz de diferentes llamadas en un único paquete. Con esto, la necesidad de ancho de banda es reducida [6].

Los parámetros del codec no cambian, luego:

- $C S I=10 \mathrm{~ms}$

- $C S S=80$ Bytes

- $V P S=160$ Bytes 
- $\quad C B R=64 \mathrm{Kbps}$

- $\operatorname{VPS}(\mathrm{ms})=20 \mathrm{~ms}$

- $P P S=50$

Para la primera llamada,

$$
T P S=L 2+I P+U D P+L 5+V P S
$$

Donde L2, IP, UDP y VPS son los mismos, pero L5 cambia ya que se utilizara IAX, cuyo tamaño de cabecera es de 4 Bytes [6].

$$
\begin{aligned}
\text { TPS }=18 \text { Bytes } & +20 \text { Bytes }+8 \text { Bytes }+4 \text { bytes } \\
& +160 \text { bytes }
\end{aligned}
$$

- $\quad T P S=210$ Bytes $=1680$ Bits

$$
\begin{gathered}
B W=\text { TPS } x \text { PPS } \\
B W=1680 \text { Bits } \times 50
\end{gathered}
$$

- $B W=84 K b p s$

Para cada llamada adicional, se reutilizan los encabezados de las capas de enlace, red y transporte, luego:

$$
\text { TPS }=4 \text { bytes }+160 \text { bytes }
$$

- $T P S=164$ Bytes $=1312$ Bits

$$
\begin{gathered}
B W=\text { TPS } x \text { PPS } \\
B W=1312 \text { Bits } \times 50
\end{gathered}
$$

- $B W=65.6 \mathrm{Kbps}$ por cada llamada adicional.

De esta manera,

- BW30calls $=84 \mathrm{Kbps}+65.6 \mathrm{Kbps} \times 29=$ 1986.4 Kbps (Aproximadamente $2 \mathrm{Mbps}$ )

\subsection{Protocolo IAX-Codec GSM}

- $C S I=20 \mathrm{~ms}$

- $C S S=33$ Bytes
- $\quad V P S=33$ Bytes

- $C B R=13.2 \mathrm{Kbps}$

- $\operatorname{VPS}(\mathrm{ms})=20 \mathrm{~ms}$

- $P P S=50$

Para la primera llamada,

$$
\begin{aligned}
& T P S=L 2+I P+U D P+L 5+V P S \\
& \text { TPS }=18 \text { Bytes }+20 \text { Bytes }+8 \text { Bytes }+4 \text { bytes } \\
& +33 \text { bytes } \\
& \text { - } T P S=83 \text { Bytes }=664 \text { Bits } \\
& B W=T P S x P P S \\
& B W=664 \text { Bits } x 50
\end{aligned}
$$

- $B W=33.2 \mathrm{Kbps}$

Para cada llamada adicional,

TPS $=4$ bytes +33 bytes

- $\quad T P S=37$ Bytes $=296$ Bits

$$
\begin{gathered}
B W=T P S \times P P S \\
B W=296 \text { Bits } x 50
\end{gathered}
$$

- $B W=14.8 \mathrm{Kbps}$

- $\quad$ BW30calls $=33.2 \mathrm{Kbps}+14.8 \mathrm{Kbps} \times 29=$ 462.4 Kbps (Menos de $512 \mathrm{Kbps}$ )

La tabla 2 muestra una síntesis de los resultados de los cálculos de uso de ancho de banda obtenidos en el trabajo anterior, al utilizar codecs de audio G.711 y GSM, para SIP e IAX (30 llamadas simultáneas): 
Tabla 2. Resultados teóricos para el uso de BW.

\begin{tabular}{|c|c|c|}
\hline PROTOC. & CODEC & $\begin{array}{c}\text { BW TEÓR. } \\
\text { REQUERIDO }\end{array}$ \\
\hline SIP & G.711 & $5232 \mathrm{Kbps}$ \\
\hline SIP & GSM & $2184 \mathrm{Kbps}$ \\
\hline IAX & G.711 & $1986.4 \mathrm{Kbps}$ \\
\hline IAX & GSM & $462.4 \mathrm{Kbps}$ \\
\hline
\end{tabular}

Fuente: elaboración propia

\section{PRUEBAS Y RESULTADOS}

El montaje físico para la implementación y el análisis de tráfico consistió en simular dos segmentos de red VoIP para una organización (una sede principal y una filial), comunicadas por un enlace Ethernet, tal como se muestra en la figura 2.

Con el fin de permitir el acceso remoto desde el sniffer, se habilitó el protocolo $\mathrm{SSH}$, tanto en los servidores de telefonía como en los routers Vyatta. Tanto la troncal SIP como IAX deben ser definidas en ambos servidores, con el fin de garantizar el establecimiento de tráfico de ida y vuelta.

\subsection{Generación de tráfico}

La central telefónica Asterisk puede hacer uso de varios recursos para realizar llamadas sin necesidad de configurar estaciones finales como teléfonos IP o softphones. La manera más sencilla y práctica es por medio de archivos de configuración. Esta funcionalidad es conocida como Asterisk Auto-dial [11].

Para esto, se configura un archivo de extensión *.call por llamada. De esta manera, se deben configurar 30 archivos para simular este mismo número de llamadas. Por simplicidad, se ha nombrado cada archivo haciendo referencia al número de extensión destino (101.call, 102.call, etc.). Los parámetros básicos para el archivo de llamada son:

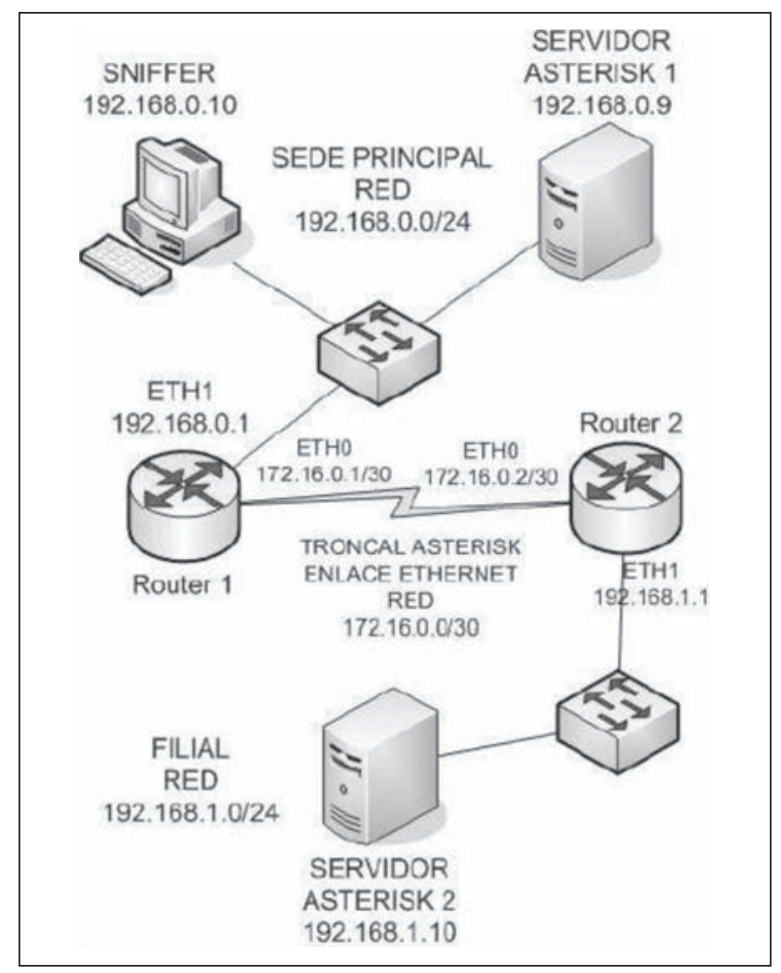

Figura 2. Mapa de red VoIP.

Fuente: elaboración propia

- Channel: corresponde al canal por el cual se va a direccionar la llamada (en este caso SIP o IAX); se debe indicar igualmente la troncal de comunicación y la extensión real de destino.

- Context: corresponde a la sección del plan de discado que recibirá la llamada.

- Extensión: corresponde a la extensión simbólica a la que se marca localmente, declarada en el contexto superior.

- Priority: indica cuál es la primera acción a realizar cuando se efectúa la llamada. Ejemplo:

Channel: SIP/TRUNK_SIP/110

Context: default

Extension: $\mathrm{s}$

Priority: 1 


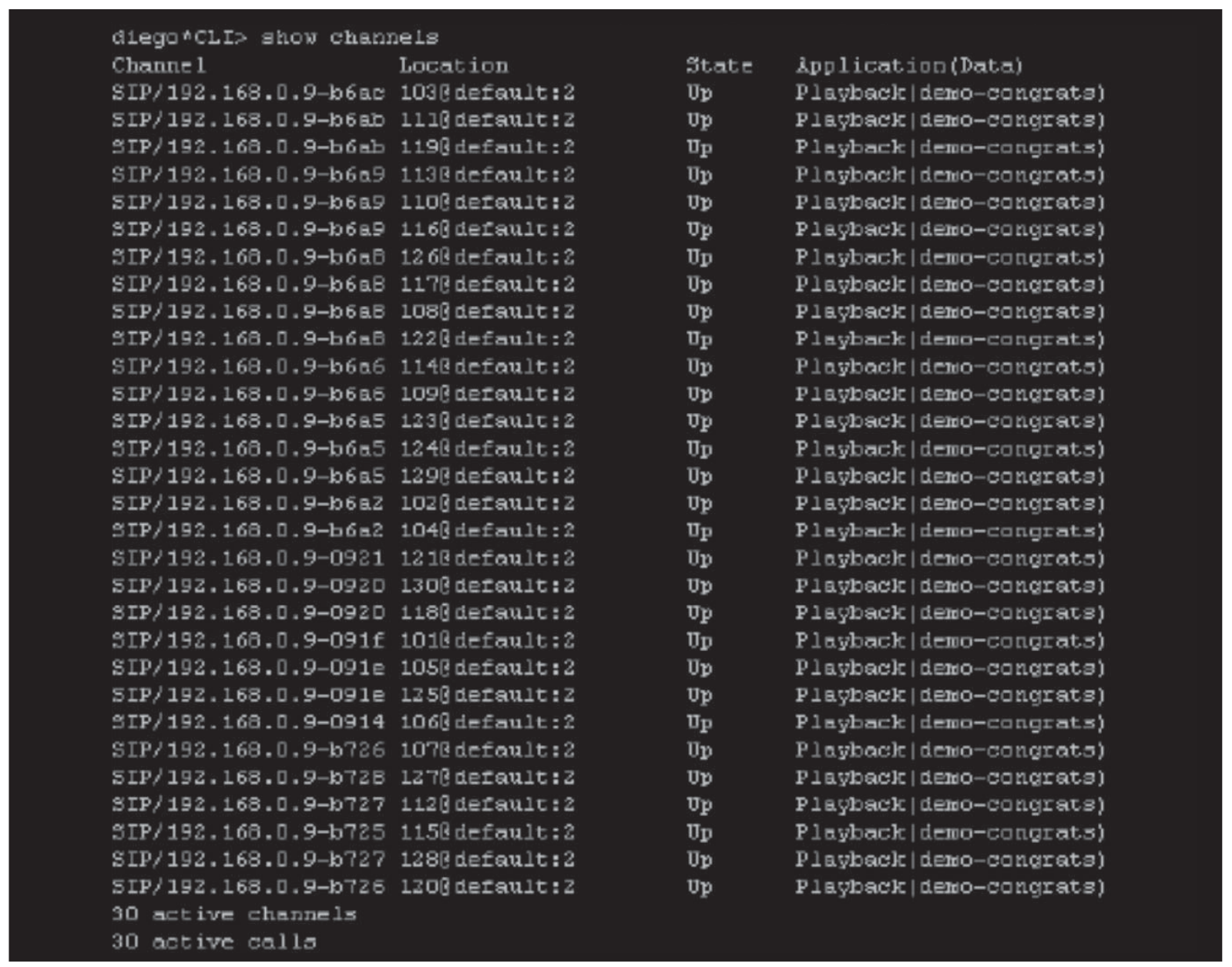

Figura 3. 30 llamadas SIP simultáneas.

Fuente: elaboración propia

Los archivos se deben ubicar en el directorio /var/ spool/asterisk/outgoing, pero deben crearse en una ubicación diferente, por ejemplo: /var/spool/ asterisk/tmp. Tan pronto sean movidos (no copiados) a /var/spool/asterisk/outgoing, la central los reconoce y, de acuerdo con los parámetros descritos en ellos, se efectúa la llamada. De acuerdo con la configuración del plan de discado, cuando el receptor contesta se ejecuta una grabación (democongrats), el cual será el flujo de media de cada una de las conversaciones.

La figura 4 muestra las llamadas simultáneas a las extensiones SIP e IAX configuradas en el servidor Asterisk 2, las cuales viajan por las troncales.

\subsection{Análisis de tráfico}

Para dicho análisis se utilizaron dos herramientas de software: Wireshark (para SIP) y Unsniff (para IAX).

\subsubsection{Procedimiento general}

Para este análisis se realizan los siguientes pasos: 
diegoncLI> show channe $1 \mathrm{~s}$

Channel Location

Idx2/192 . 168.0.9:456 2278derau1t: 2

Isx2/192.160.0.9:456 207Rdefault:2

Isxe/193.168.0.9:956 203RdeIau1t:a

I $132 / 192$ - $160.0 .9: 456$ 213Bdefault: 2

IAx2/192.168.0.9:456 211Rdefault:3

I lxz/192.160.0.9:456 220ßdefault: 2

IAX2/192.168.0.9:456 229[default :

I $\lambda$ z / $192.168 .0 .9: 456$ 2068derau $1 \tau: 2$

Isxe/192.168.0.9:456 223Edefault:2

IAr2/192.168.0.9:456 226Bderaulc : 2

I $\mathrm{s} 2 / 192.168 .0 .9: 456$ 2158default:2

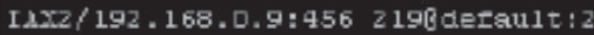

Is:2/192.169.0.9:456 210Rdefault:2

Idx2/192.168.0.9:456 2048deIsu1t:2

Is:2/192.160.0.9:456 209Rdefault:2

Isxz/193.168.0.9:956 224ldeIau1t:z

I lxz/192.160.0.9:456 212Bdefault: 2

Ibx2/192 . 168.0.9:456 214Rdefault : 2

I lis: / 192 . $160.0 .9: 456$ 202Bdefault : 2

I hx2/192 . 168.0.9:456 225Rdefault :

I $x z 2 / 192.168 .0 .9: 456$ 221Bderault:

Is:2/192.168.0.9:456 200Edefault:2

IArz/192.168.0.9:456 218Bderault:2

Is:2/192.168.0.9:456 201Bdefault :2

Isxz/192 . 168.0.9:456 2178 desau1t: 2

Is:2/192,168.0.9:456 230Rdefault:2

IAx2/192 . 168.0.9:956 2208deIau1t:2

Is:2/192,160.0.9:456 216Rdefault ;

Isxz/193 . 168.0.9:956 2zzedeIau1t:a

I $\lambda z 2 / 192$. $160.0 .9: 456$ 205Bdefault : 2

30 active channels

so active calls
Avplication (Datal

playback (dero-concreats)

Pl avback (demo-congr ats)

Playback (dero-concrata)

P layback (demo-congrats)

Playback (deroo-concreata)

P layback (demo-congrats)

Plapback (dero-congrata)

Playbac x (dero-congrets)

Plavback (deno-congrats)

Playbacx (dero-congracs)

Playback (devo-congr ats)

playbac $x$ (dero-congrats)

Playback (dewo-congr ats)

playback (dero-concrets)

Playback (demo-congr ats)

playback (deroo-congrata)

Playback (demo-congrats)

plapback (dero-congrata)

Playback (demo-congrats)

Plawback (dero-congrata)

Playbac x (dero-congrats)

Plapback (dero-congrats)

Playbacx (deroo-congrats)

Playback (devo-congr ats)

Playback (deroo-congrats)

Playback (dero-congr ats)

playback (deroo-concrets)

Playback (demo-congr ats)

playback (dero-congrata)

Playback (demo-congrats)

Figura 4. 30 llamadas IAX simultáneas.

Fuente: elaboración propia

- Instalación de vyatta system en los PCs que actuarán como routers [12].

- Configuración de las interfaces y las rutas estáticas en los Vyatta [12].

- Instalación del sistema operativo Centos 5.2 en los servidores Asterisk 1 y Asterisk 2 [13].

- Instalación de Asterisk PBX en los servidores 1 y 2 [14], [15].

- Instalación de Wireshark y Unsniff en la maquina sniffer [16], [17].

- Configuración de 30 extensiones SIP (101130) y 30 extensiones IAX (201-230) en el servidor Asterisk 2 (editando los archivos sip. conf e iax.conf, respectivamente)

- Configuración del plan de discado en el servidor Asterisk 2, de tal forma que, al recibir una llamada, se ejecute una grabación predeterminada que simulará el flujo de media (editando el archivo extensions.conf).

- Se crean 60 archivos con extensión .call en la ubicación /var/spool/asterisk/tmp en el servidor Asterisk 1, 30 para SIP (101.call - 130. call) y 30 para IAX (201.call - 230.call) con los cuales se generarán las llamadas desde el servidor 1 hasta el 2 [11]. 
[rootedsTeRI9R1 asterisk] H asterisk - $[x$ "sid show peers"

Noan/ uaernome

TRUNK SIP/TRTNR SIP

Host

192.160 .1 .9

Dyn Nat ACL Port

5060
Retatus

OR $(1 \mathrm{~ms})$

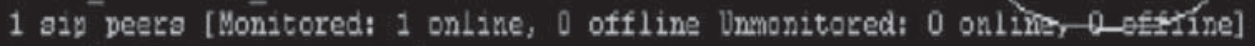

[roat(isTeRIgR1 asterisk] Asterisk -rx "iax2 shov peers"

Name/laername Hoat Kaak Port

$\begin{array}{lllll}\text { TRUNK I IX } & 192.168 .1 .9 & \text { |9| } & 255.255 .255 .255 & 4569\end{array}$

1 iax $\overline{2}$ yeers [ 1 online, 0 offline, 0 unronitored]

Figura 5. Comparación de retardo para SIP e IAX.

Fuente: elaboración propia

- Configuración del plan de discado en el servidor 1 , creando un contexto, extensión y prioridad simbólicos por donde se van a lanzar las llamadas al servidor Asterisk 2.

- Ejecución del software Asterisk en ambas centrales telefónicas. La figura 5 muestra los respectivos tiempos de retardo para las troncales SIP e IAX.

\subsubsection{Análisis de SIP con Wireshark}

- Se inicia una captura en Wireshark, verificando la correcta elección de la interfaz de red por donde se deben capturar los datos.

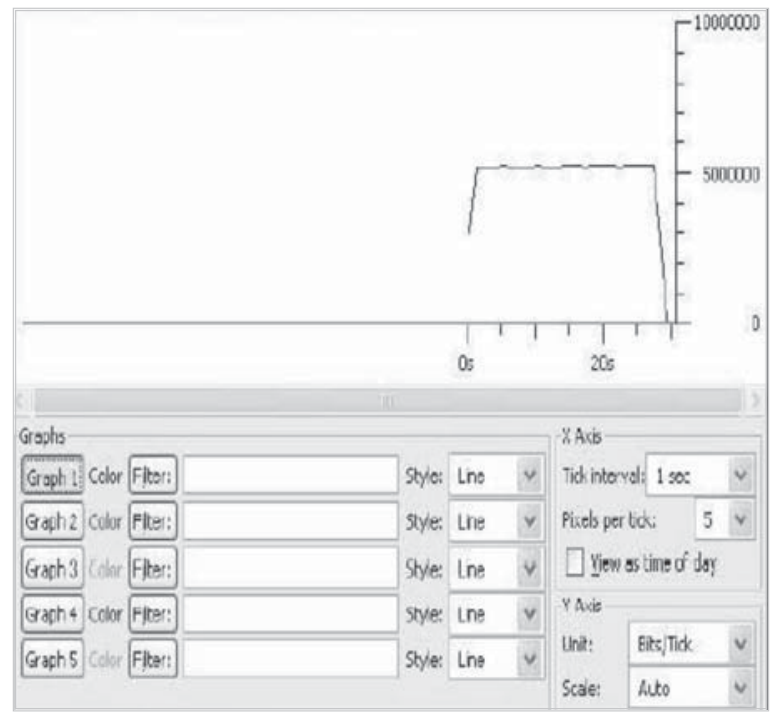

Figura 6. BW SIP, G.711, 30 llamadas.

Fuente: elaboración propia
- Se deben mover (no copiar) los archivos .call (101.call - 130.call) al directorio /var/spool/ asterisk/outgoing (debe mantenerse un backup de estos archivos para su uso posterior).

- Con el comando "show channels" en la consola del Asterisk, es posible visualizar, en tiempo real, los 30 canales, tal como se muestra en la figura 3.

- Cuando las llamadas finalicen, se detiene la captura de Wireshark y se guarda.

La figura 6, la figura 7, la figura 8 y la 9 , muestran los resultados del análisis de uso de ancho de

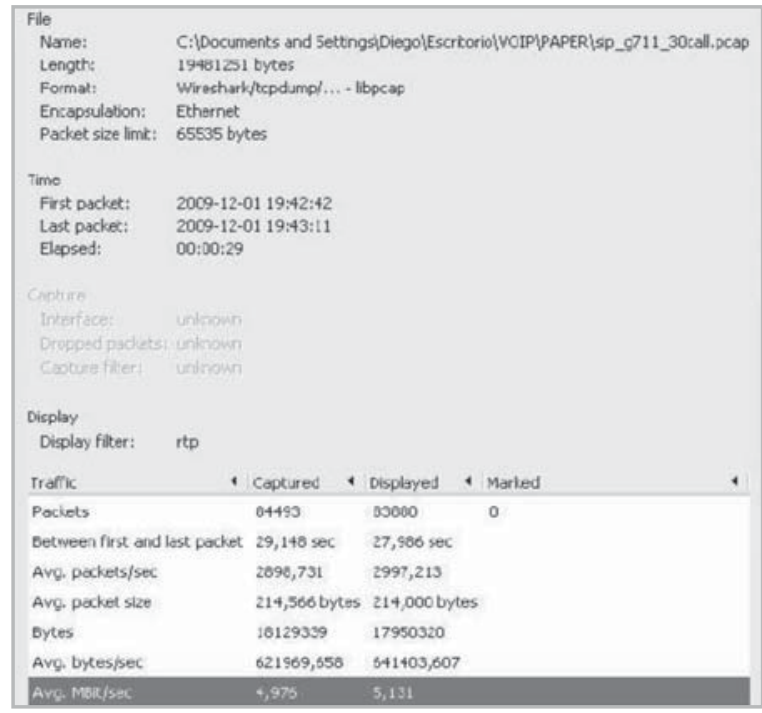

Figura 7. Resumen SIP, G.711, 30 llamadas.

Fuente: elaboración propia 


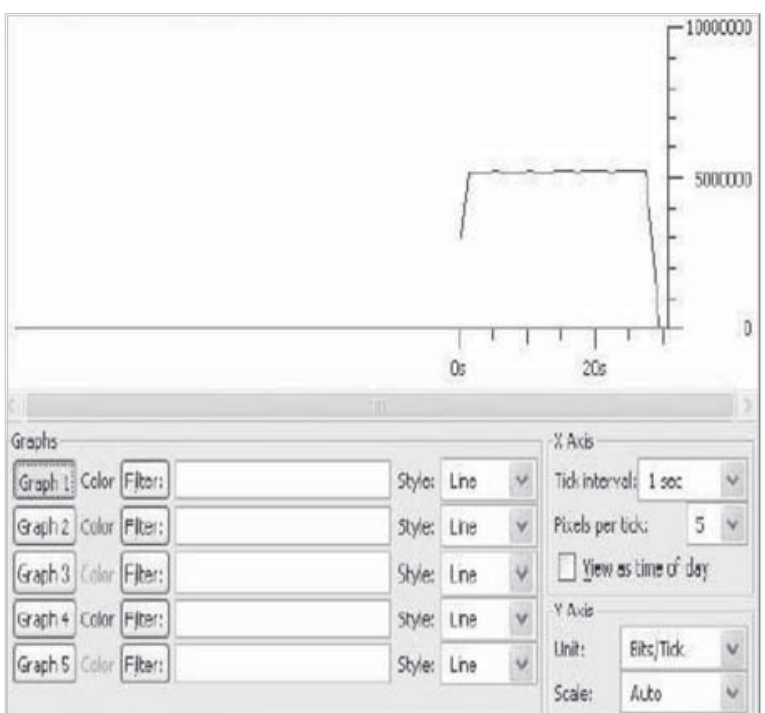

Figura 8. BW SIP, GSM, 30 llamadas.

Fuente: elaboración propia

banda para 30 llamadas, con los codecs G.711 y GSM, respectivamente.

De acuerdo con la figura 7, BW30calls $=5.131$ Mbps (valor promedio).

De acuerdo con la figura 9, BW30calls $=2.087$ Mbps (valor promedio).

\subsubsection{Análisis de IAX con Unsniff}

Una vez realizado el procedimiento general, se procede a realizar los siguientes pasos:

- Se inicia una captura en Unsniff.

- Se deben mover (no copiar) los archivos .call (201.call - 230.call) al directorio /var/spool/ asterisk/outgoing (debe mantenerse un backup de estos archivos para su uso posterior).

- Con el comando "show channels" en la consola del Asterisk es posible visualizar, en tiempo real, los 30 canales, tal como se muestra en la figura 3.

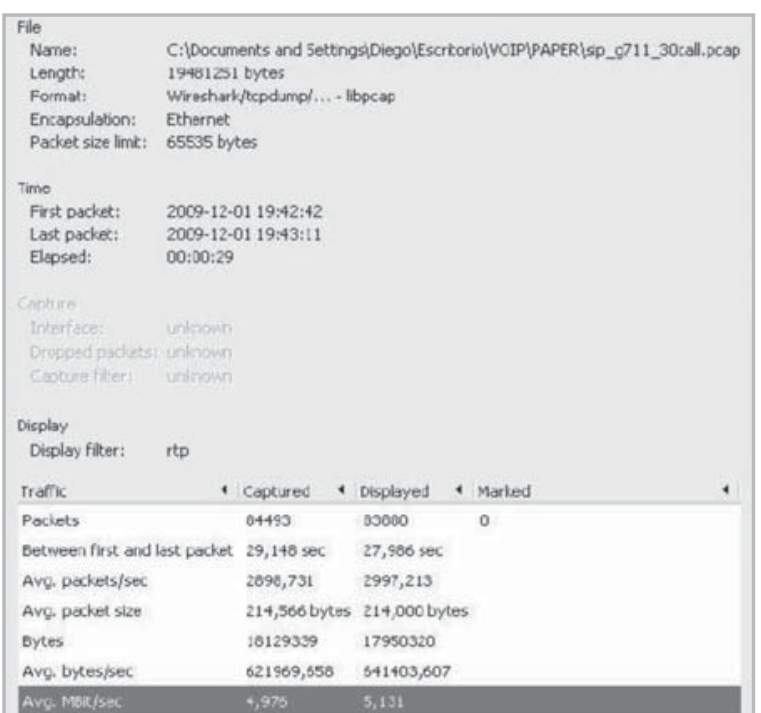

Figura 9. Resumen SIP, GSM, 30 llamadas.

Fuente: elaboración propia

- Cuando las llamadas finalicen, se detiene la captura de Unsniff y se guarda.

El analizador Unsniff presenta una limitación: el $B W$ máximo que captura son $200 \mathrm{Kbps}$; por esta razón, para calcular el $\mathrm{BW}$ correspondiente a 30 llamadas, se procede a capturar paquetes, inicialmente para una llamada, luego para dos y posteriormente para tres llamadas.

Las figuras 10 y 11 muestran los resultados del análisis de uso de ancho de banda para 30 llamadas, con los codecs G.711 y GSM, respectivamente (con G.711, el BW para tres llamadas es mayor que $200 \mathrm{Kbps}$, por lo que no se grafica).

De acuerdo con la figura 10, el BWlcall es de aproximadamente $78 \mathrm{Kbps}$ (valor promedio).

De acuerdo con la figura 11, el BW2calls es de aproximadamente $150 \mathrm{Kbps}$ (valor promedio). Esto quiere decir que, a partir de la segunda llamada, cada una consume un $B W$ de aproximadamente $150-78=72 \mathrm{Kbps}$. Luego, BW30calls $=$ $78 \mathrm{Kbps}+72 \mathrm{Kbps}$ x $29=2166 \mathrm{Kbps}$ 


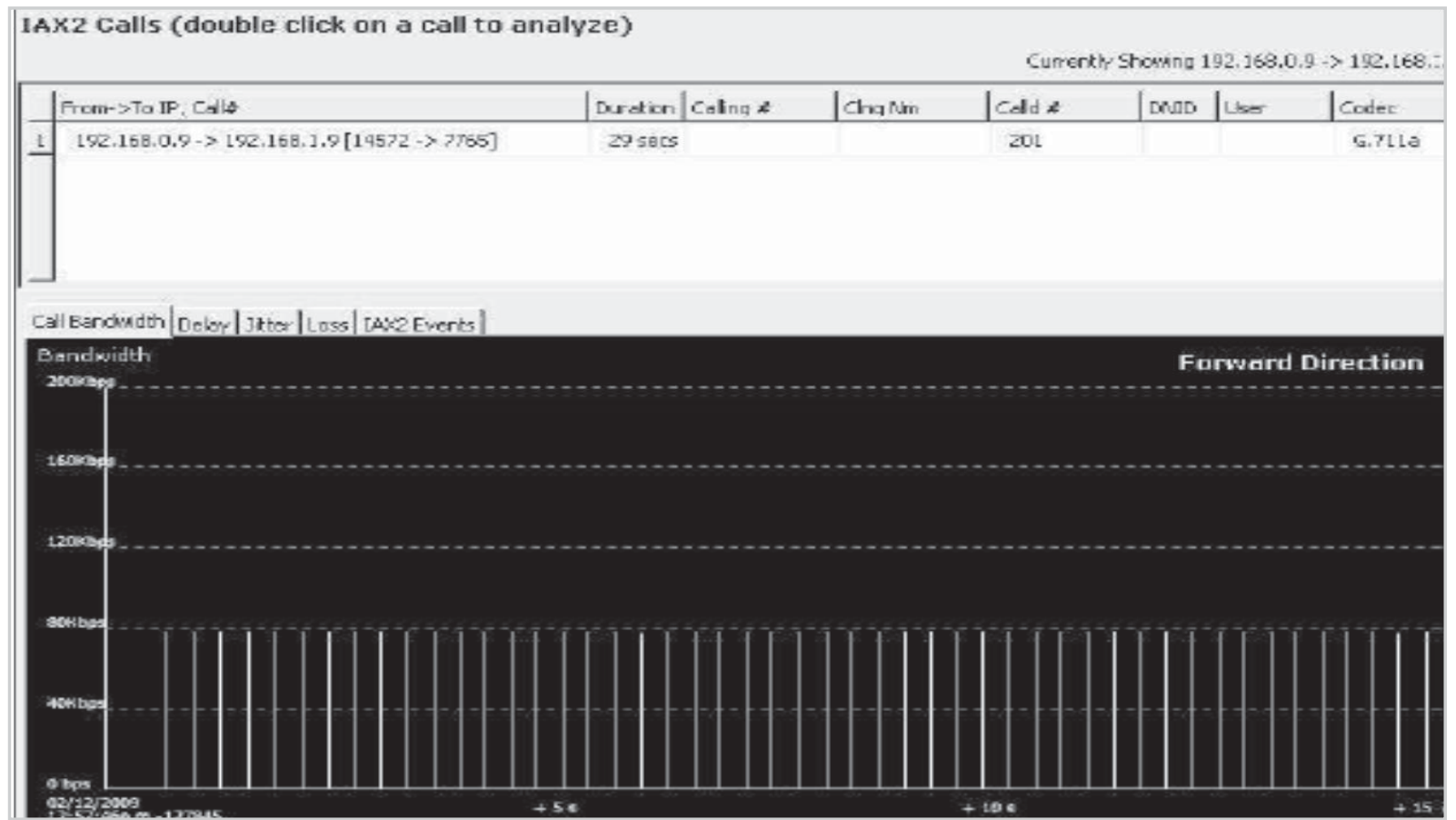

Figura 10. BW IAX, G.711, 1 llamada.

Fuente: elaboración propia

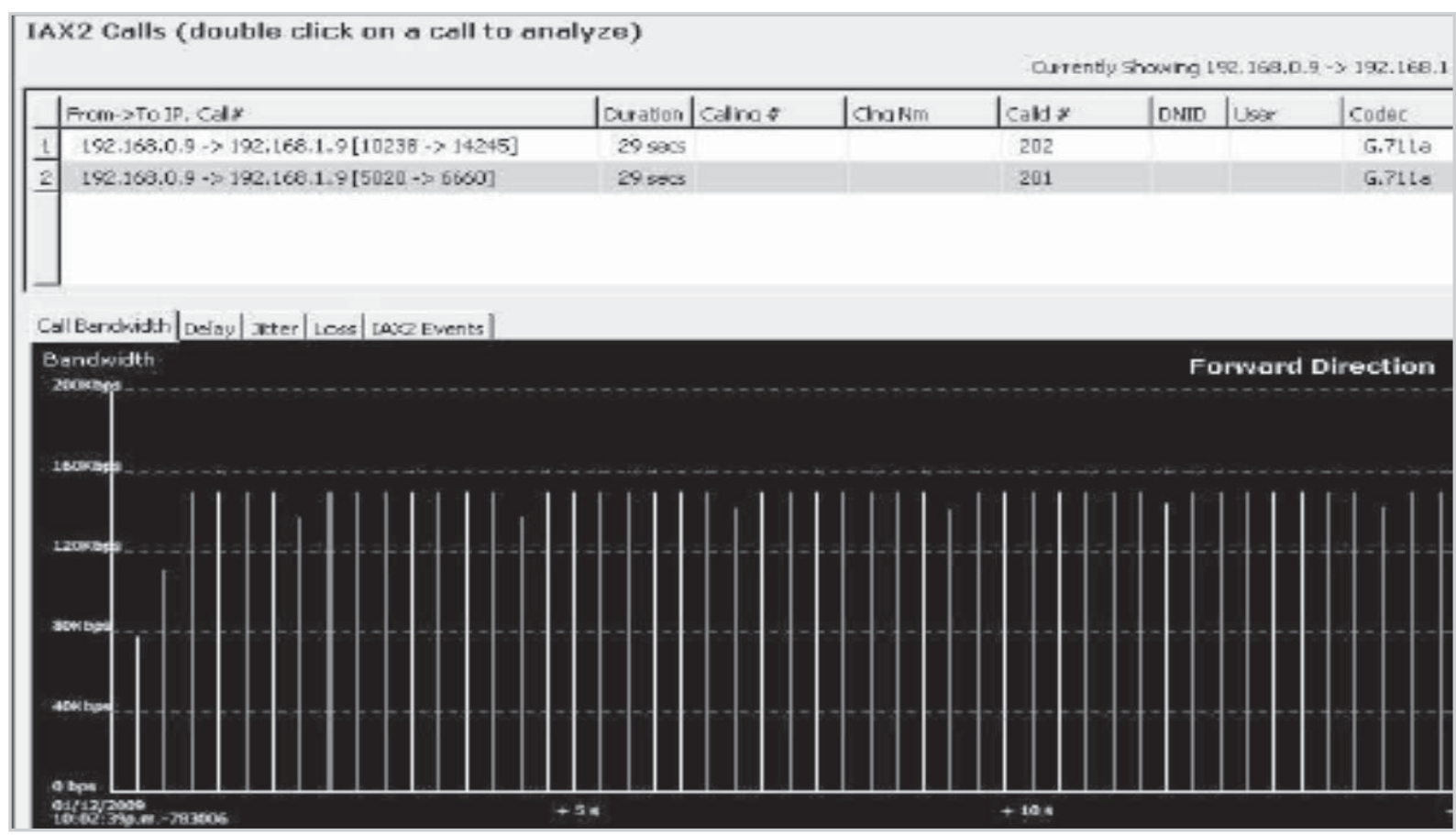

Figura 11. BW IAX, G.711, 2 llamadas.

Fuente: elaboración propia 


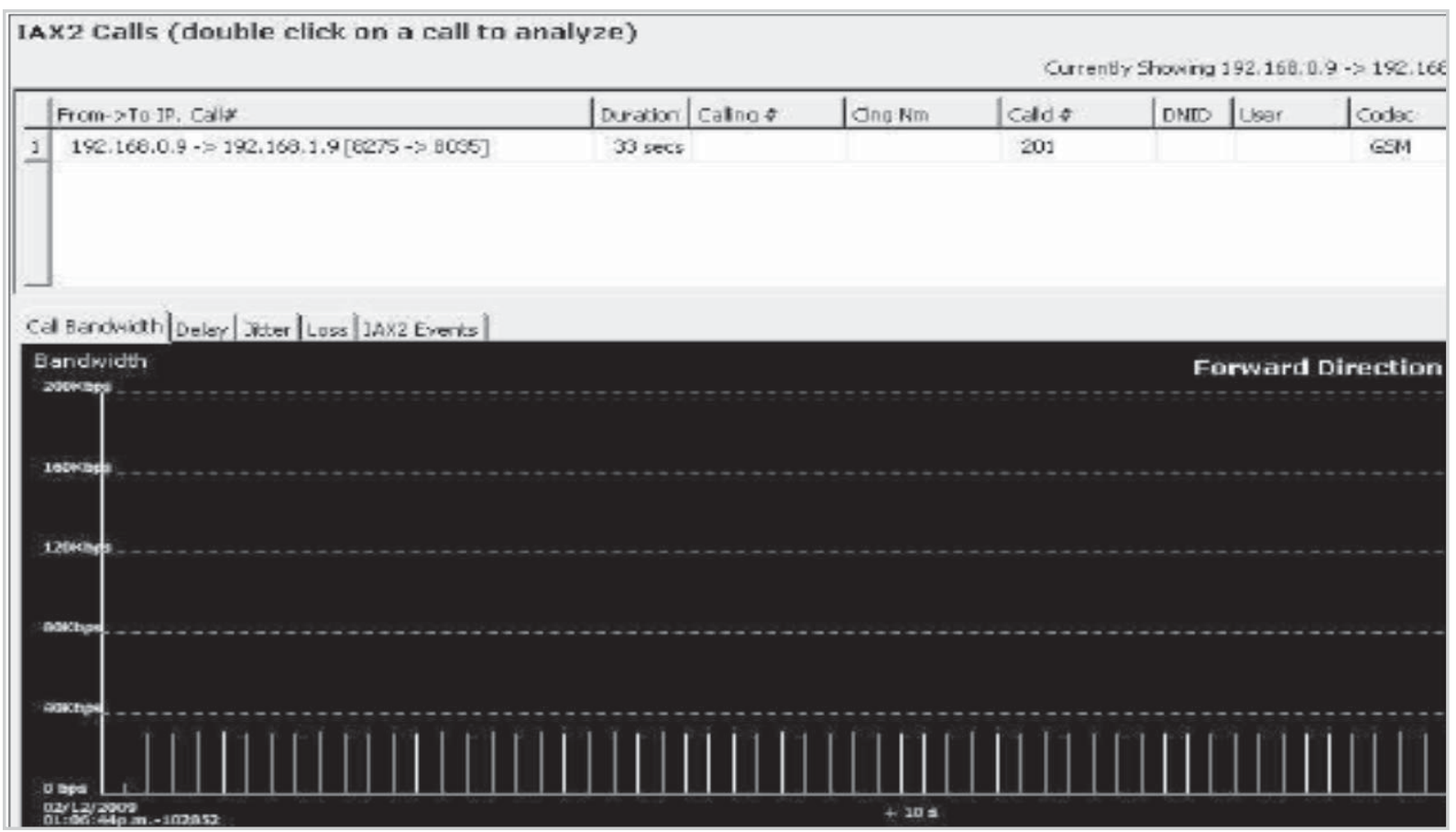

Figura 12. BW IAX, GSM, 1 llamada.

Fuente: elaboración propia

De acuerdo con la figura 12, BWlcall es de aproximadamente $30 \mathrm{Kbps}$ (valor promedio).

De acuerdo con la figura 13, BW2calls es de aproximadamente $48 \mathrm{Kbps}$ (valor promedio).

De acuerdo con la figura 14, BW3calls es de aproximadamente $75 \mathrm{Kbps}$ (valor promedio). Esto quiere decir que, a partir de la segunda llamada, cada una consume un $B W$ de aproximadamente $48-30=18 \mathrm{Kbps}$. Luego, $B W 30$ calls $=30$ Kbps +18 Kbps x $29=552$ Kbps.

Tabla 3. Resultados uso de BW.

\begin{tabular}{|c|c|c|c|}
\hline PROTOC. & CODEC & BW TEÓR. & BW REAL \\
\hline SIP & G.711 & $5232 \mathrm{Kbps}$ & $5131 \mathrm{Kbps}$ \\
\hline SIP & GSM & $2184 \mathrm{Kbps}$ & $2087 \mathrm{Kbps}$ \\
\hline IAX & G.711 & $1986.4 \mathrm{Kbps}$ & $2166 \mathrm{Kbps}$ \\
\hline IAX & GSM & $462.4 \mathrm{Kbps}$ & $552 \mathrm{Kbps}$ \\
\hline
\end{tabular}

Fuente: elaboración propia
La tabla 3 muestra, a partir de los cálculos teóricos de la tabla 2 y los resultados de los analizadores en el montaje real, la comparación de valores de utilización de BW para la troncal VoIP.

\section{CONCLUSIONES}

La central telefónica configurada con protocolo SIP y codec G.711, presenta el mayor consumo de ancho de banda; esto se debe, en gran parte, al tamaño de la cabecera del protocolo SIP y a la baja tasa de compresión del codec G.711. Esta configuración, sin embargo, presenta una muy buena calidad de voz, por lo que es ideal para PBX con un nivel de tráfico relativamente bajo.

La central telefónica configurada con protocolo IAX y codec GSM, presenta el menor consumo de ancho de banda, debido a la reutilización de las cabeceras de la capa de red y transporte y a la alta tasa de compresión de GSM con respecto a 


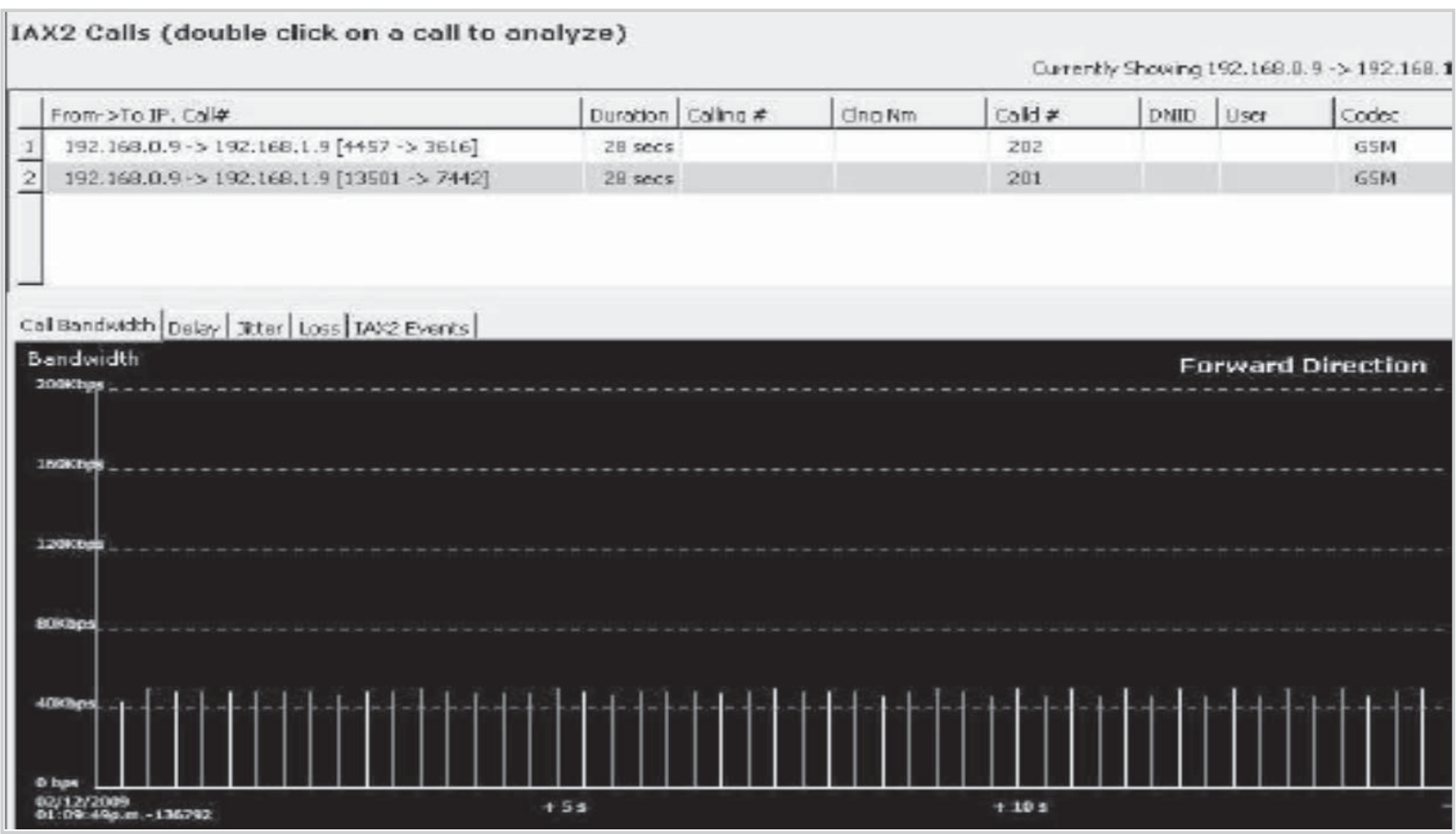

Figura 13. BW IAX, GSM, 2 llamadas.

Fuente: elaboración propia

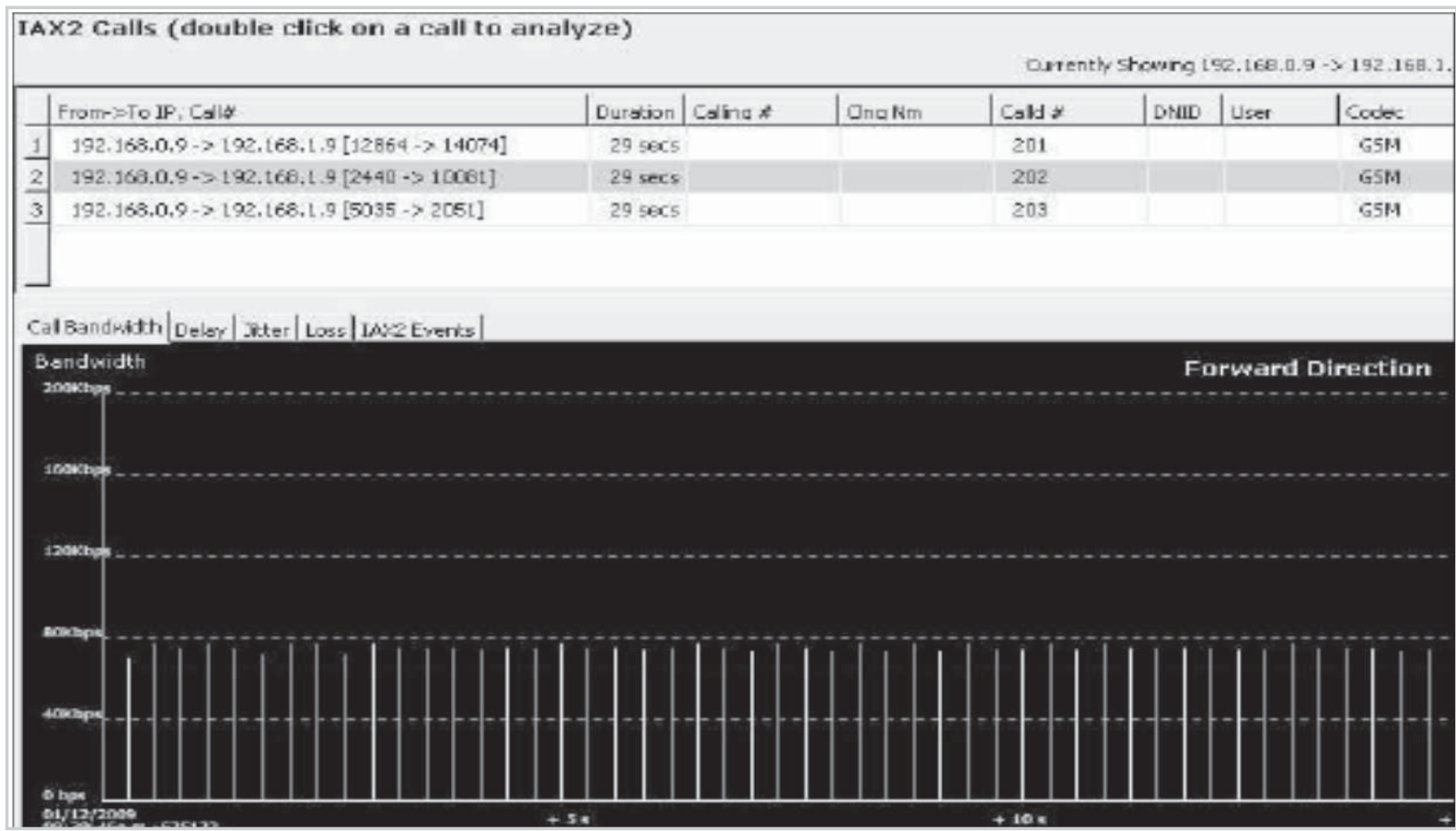

Figura 14. BW IAX, GSM, 3 llamadas.

Fuente: elaboración propia 
G.711. Esta configuración, presenta una aceptable calidad de voz pero, en ciertos momentos de alto tráfico, pueden presentarse distorsiones.

La central telefónica configurada con protocolo IAX y codec G.711 es ideal para centrales Asterisk o compatibles con IAX, cuyo nivel de tráfico sea relativamente alto, ya que presenta una buena calidad de voz pero no requiere de un ancho de banda tan elevado.

La central telefónica configurada con protocolo SIP y codec GSM es ideal para centrales no compatibles con IAX, presentando las mismas bondades que la configuración anterior.

El protocolo IAX fue desarrollado por sus creadores con el objetivo de minimizar el efecto producido por una serie de inconvenientes que se encontraron al utilizar SIP en VoIP, como el uso de diferentes puertos para señalización y transferencia de media.
Por medio de IAX, en modo trunked, es posible disminuir el ancho de banda de las conversaciones, por lo que en enlace entre dos gateways pueden efectuarse un mayor número de llamadas simultáneas.

SIP sigue siendo el protocolo más robusto y utilizado por la gran mayoría de fabricantes de hardware y software; es un protocolo de propósito general, lo que quiere decir que puede implementarse no solamente para audio o video, sino para cualquier tipo de datos. IAX, en cambio, es orientado a multimedia, hasta hace poco se estandarizó y es por ello que no se encuentra en muchos dispositivos existentes en el mercado.

De acuerdo con la figura 5, la comunicación entre las dos centrales telefónicas a través de la troncal SIP presenta menos retardo $(1 \mathrm{~ms})$ que por medio de la troncal IAX (6ms).

\section{REFERENCIAS}

[1] R. Gallego, Transmisión de Audio, QoS en redes de nueva generación, Departamento de Ingeniería Electrónica y Comunicaciones: Universidad de Zaragoza, 2005.

[2] Cisco Systems, Bandwidth per call, (2011), [online]. Available: http://www.cisco.com/ en/US/tech/tk652/tk698/technologies tech_note09186a0080094ae2.shtml\#topic

[3] F. E. Goncalves, Asterisk PBX guía de la configuración, (Tercera generación), [1a Ed.], Rio de Janeiro 2007

[4] T. Abbasi, S. Prasad, N. Seddigh, and I. Lambadaris, "A comparative study of the sip and iax voip protocols", in Electrical and Computer Engineering, 2005. [Ca- nadian Conference on], Saskatoon, Sask, May, 2005, pp. 179-183.

[5] K. S. Chava and J. How, "Integration of Open Source and Enterprise IP PBXs", in Testbeds and Research Infrastructure for the Development of Networks and Communities, 2007. [TridentCom 2007. 3rd International Conference on], Lake Buena Vista, FL, 21 al 23 May, 2007, pp. 1-6.

[6] M. Spencer, B. Capouch, and E. Guy, RFC 5456 for IAX, 2011, [online]. Available: http://www.rfc-editor.org/authors/rfc5456. txt.

[7] J. Rosenberg, et al, RFC 3261 - SIP: Session Initiation Protocol, June, 2002 


\section{revisión}

[8] G. Gorrotxategui y I. Baz, "Curso sobre VoIP y Asterisk Modulo 1", en IRONTEC, 2002, [en linea]. Disponible: http://www. irontec.com.

[9] P. Montoro, and E. Casilari; "A Comparative Study of VoIP Standards with Asterisk", in Digital Telecommunications, 2009. ICDT '09. [Fourth International Conference on], Colmar, Francia, July, 2009, pp. 1-6.

[10] J. T. Da Loligo, (2011), Cálculo de ancho de banda para IAX modo trunked, [en linea]. Disponible: http://www.voipinfo.org/ wiki/wiki-Asterisk+bandwidth+iax2.

[11] Asterisk Auto-dial, (2011), [en línea]. Disponible: http://www.voipinfo.org/wiki/ view/Asterisk+auto-dial+out.

[12] Vyatta Open Networking, (2011), Software, productos y manuales de instalación y configuración, [en línea]. Disponible: http:// www.vyatta.com/.

[13] Instalación CentOS 5.1 para centrales telefónicas Open Source Asterisk
PBX, (2011), [en línea]. Disponible: http://192.168.222.5/wiki/index.php/Instalacion_CentOS_5.1_para_Asterisk_-GNU

[14] Instalación y configuración de Asterisk PBX en Ubuntu Server, (2011), [en línea]. Disponible: http://www.voipinfo.org/wiki/ view/Instalaci $\%$ C3\%B3n+de+Asterisk+en + Ubuntu+Server+Paso+a+Paso.

[15] Instalación y configuración de Asterisk sobre CENTOS 5.2, (2011), [en línea]. Disponible: http://wiki.millenium.com.co/wiki/ index.php/Instalacion_Asterisk_1.4.X.

[16] Protocol Analyzer for IAX2, (2011), [en línea]. Disponible: http://www.unleashnetworks.com/resources/articles/72-asteriskcall-analyzer-for-iax $2 . \mathrm{html}$.

[17] Análisis de paquetes IAX en la herramienta Wireshark, (2011), [en línea]. Disponible: http://wiki.wireshark.org/IAX2. 